Ferrata Storti Foundation

\title{
Early growth response 1 regulates hematopoietic support and proliferation in human primary bone marrow stromal cells
}

Haematologica 2020

Volume 105(5):1206-1215

\section{Correspondence: \\ STEFAN SCHEDING \\ stefan.scheding@med.lu.se \\ Received: January 14, 2019. \\ Accepted: July 19, 2019. \\ Pre-published: August 1, 2019.}

doi:10.3324/haematol.2019.216648

Check the online version for the most updated information on this article, online supplements, and information on authorship \& disclosures: www.haematologica.org/content/105/5/1206

(C)2020 Ferrata Storti Foundation

Material published in Haematologica is covered by copyright. All rights are reserved to the Ferrata Storti Foundation. Use of published material is allowed under the following terms and conditions:

https://creativecommons.org/licenses/by-nc/4.0/legalcode. Copies of published material are allowed for personal or internal use. Sharing published material for non-commercial purposes is subject to the following conditions:

https://creativecommons.org//icenses/by-nc/4.0/legalcode, sect. 3. Reproducing and sharing published material for commercial purposes is not allowed without permission in writing from the publisher.

\author{
Hongzhe $\mathrm{Li}^{1,2}$ Hooi-Ching Lim, ${ }^{1,2}$ Dimitra Zacharaki, ${ }^{1,2}$ Xiaojie Xian, ${ }^{2,3}$ \\ Keane J.G. Kenswil, ${ }^{4}$ Sandro Bräunig, ${ }^{1,2}$ Marc H.G.P. Raaijmakers, ${ }^{4}$ \\ Niels-Bjarne Woods, ${ }^{2,3}$ Jenny Hansson, ${ }^{1,2}$ and Stefan Scheding ${ }^{1,2,5}$
}

${ }^{1}$ Division of Molecular Hematology, Department of Laboratory Medicine, Lund University, Lund, Sweden; ' 2 Lund Stem Cell Center, Department of Laboratory Medicine, Lund University, Lund, Sweden; ' ${ }^{2}$ ivision of Molecular Medicine and Gene Therapy, Department of Laboratory Medicine, Lund University, Lund, Sweden; ${ }^{4}$ Department of Hematology, Erasmus MC Cancer Institute, Rotterdam, the Netherlands and ${ }^{5}$ Department of Hematology, Skåne University Hospital Lund, Skåne, Sweden

\section{ABSTRACT}

$\mathrm{H}$ uman bone marrow stromal cells (BMSC) are key elements of the hematopoietic environment and they play a central role in bone and bone marrow physiology. However, how key stromal cell functions are regulated is largely unknown. We analyzed the role of the immediate early response transcription factor EGR1 as key stromal cell regulator and found that EGR1 was highly expressed in prospectivelyisolated primary BMSC, down-regulated upon culture, and low in noncolony-forming $\mathrm{CD} 45^{\text {neg }}$ stromal cells. Furthermore, EGR1 expression was lower in proliferative regenerating adult and fetal primary cells compared to adult steady-state BMSC. Overexpression of EGR1 in stromal cells induced potent hematopoietic stroma support as indicated by an increased production of transplantable $\mathrm{CD} 34^{+} \mathrm{CD} 90^{+}$hematopoietic stem cells in expansion co-cultures. The improvement in bone marrow stroma support function was mediated by increased expression of hematopoietic supporting genes, such as VCAM1 and CCL28. Furthermore, EGR1 overexpression markedly decreased stromal cell proliferation whereas EGR1 knockdown caused the opposite effects. These findings thus show that EGR1 is a key stromal transcription factor with a dual role in regulating proliferation and hematopoietic stroma support function that is controlling a genetic program to co-ordinate the specific functions of BMSC in their different biological contexts.

\section{Introduction}

Hematopoietic stem cell (HSC) niches are specialized local tissue microenvironments that maintain and regulate HSC. Aside from being skeletal stem cells with multilineage differentiation capacity, bone marrow mesenchymal stromal cells (BMSC) are essential constituents of the HSC niche. Despite this key role in hematopoiesis, and despite recent progress in the identification of primary BMSC, ${ }^{1,2}$ little is known about how proliferation, differentiation and hematopoietic support functions of these important niche cells are regulated.

We therefore approached the identification of potential BMSC regulators by investigating specific gene expression of highly-purified primary BMSC that were prospectively isolated from human bone marrow samples using recently-identified surface marker combinations. ${ }^{1}$ Previously reported array-based comparative gene expression analysis showed high expression of early growth response 1 (EGR1) in primary BMSC compared to non-colony-forming cells (for details see Online Supplementary Table S1 in $\mathrm{Li}$ et al. ${ }^{1}$ ). Interestingly, EGR1 is an important regulator of different cellular processes ${ }^{3.5}$ and has been identified as a cell-intrinsic regulator of HSC proliferation and mobilization. ${ }^{6}$ Furthermore, data from knockout mice indicated a role of EGR1 on bone formation in vivo. These findings suggested that 
EGR1 might also have a role in human primary nonhematopoietic bone marrow stromal cells.

We therefore investigated the role of EGR1 in human BMSC and found that EGR1 was highly expressed in steady-state primary BMSC compared to regenerating adult and fetal BMSC. Overexpression of EGR1 increased BMSC hematopoietic stroma support function and inhibited cell proliferation, whereas decreased EGR1 expression caused the opposite effects. Our data thus indicate a key dual role of EGR1 in BMSC regulation, which has important implications for stroma repair and replacement approaches, and possibly also for the understanding of important developmental aspects of hematopoietic stroma formation.

\section{Methods}

\section{Human bone marrow and cord blood cells}

Human bone marrow (BM) cells were collected at the Hematology Department, University of Lund, Sweden, from consenting healthy donors by aspiration from the iliac crest. Cord blood (CB) samples were obtained from consenting donors at the maternity wards at Lund, Helsingborg and Malmö Hospitals, Sweden. BM aspirates from patients with acute myeloid leukemia (AML), BM controls, and fetal long bones were obtained with informed consent at the Erasmus Medical Center, the Netherlands, as described previously. ${ }^{8}$ The processing of BM mononuclear cells (BM-MNC), isolation and characterization of primary bone marrow stroma cells (BMSC), and generation of cultured stroma cells including EGR1 over-expressing and knockout cells from sorted CD $45^{-} \mathrm{CD} 271^{+} \mathrm{CD} 140 \mathrm{a}$ BMSC, is provided in the Online Supplementary Appendix: experimental procedures. The use of human samples was approved by the corresponding Institutional Review Board of the University of Lund and the Erasmus Medical Center, respectively, in accordance with the Declaration of Helsinki.

\section{Co-culture of cord blood CD34+ cells with stromal cells}

FACS-sorted $\mathrm{GFP}^{+}$stromal cells (EGR1 knockdown, EGR1 over-expressing cells, and corresponding controls) were plated as adherent feeder cells into 96-well plates at 10,000 cells per well and cultured in MSC medium for three days. Medium was then removed and 5,000 $\mathrm{CB} \mathrm{CD} 34^{+}$cells were added in serum-free expansion medium (SFEM, STEMCELL Technologies) supplemented with or without stem cell factor, thrombopoietin, and FLT-3 ligand (all at $25 \mathrm{ng} / \mathrm{mL}$, STF25) (Online Supplementary Appendix). Expanded CB cells were harvested, counted and analyzed after four days of co-culture.

Conditioned MSC medium was prepared by replacing the MSC expansion medium with serum-free expansion medium (SFEM) and collecting the conditioned media after four days.

Transwell cultures were performed using the HTS Transwell96 System (Corning, New York, NY, USA); 10,000 BMSC in standard MSC culture medium were plated in the transwell reservoir. After three days of culture, the MSC culture medium was removed and replaced with SFEM (STF25), and 5,000 CB $\mathrm{CD}^{+} 4^{+}$cells in SFEM (STF25) were loaded into the insert (membrane pore size: $0.4 \mu \mathrm{m}$ ). After four days of co-culture, cells were harvested, counted and analyzed.

To evaluate the role of VCAM1 and CCL28 on CD34 expansion, stromal cells were pretreated with $100 \mathrm{ng} / \mathrm{mL}$ VCAM1 or CCL28 blocking antibody and control IgG, respectively, at $37^{\circ} \mathrm{C}$ for two hours followed by co-culture with $\mathrm{CD} 34^{+}$cells in either standard SFEM (STF25) or cytokine-free conditions at $37^{\circ} \mathrm{C}$ for four days.

\section{Other methods}

The details of the other methods used in this study, i.e. generation of cultured MSC, EGR1 knockdown and EGR1 overexpressing stroma cells, FACS sorting and analysis, CFU-F assays, in vitro differentiation assays, real-time polymerase chain reaction (PCR), in vivo HSC repopulation assay, CCL28 ELISA, Illumina array, RNA-seq and proteome analysis, as well as information on the deposition of gene expression and proteomics data, are all provided in the Online Supplementary Appendix: experimental procedures.

\section{Results}

\section{EGR1 is highly expressed in primary bone marrow} stromal cells

Our previous gene expression profiling data demonstrated that expression levels of EGR1 in primary CFU-F (colony-forming unit, fibroblast)-enriched lin ${ }^{-} \mathrm{CD} 45$ CD271+ ${ }^{+}$BMSC were substantially higher compared to non-colony-forming cells $\left(\operatorname{lin}^{-} \mathrm{CD} 45^{-} \mathrm{CD} 271^{-}\right) .{ }^{1}$ We therefore proceeded to investigate EGR1 expression and function in highly purified lin $^{-} \mathrm{CD} 45^{-} \mathrm{CD} 271^{-} \mathrm{CD} 140 \mathrm{a}$ (PDGFR $\alpha)^{-}$BMSC, which we have recently demonstrated as a (close to) pure population of putative BM stromal stem cells with high CFU-F frequency, typical in vitro and in vivo differentiation capacities, and potent hematopoietic stroma function. ${ }^{1}$ Expression of EGR1 was $128.9 \pm 28.4$ fold higher in lin $^{-} \mathrm{CD} 45^{-} \mathrm{CD} 271^{+} \mathrm{CD} 140 \mathrm{a}^{-} \mathrm{BMSC}$ compared to non-colony-forming cells

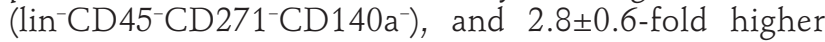
compared to $\operatorname{lin}^{-} \mathrm{CD} 45^{-} \mathrm{CD} 271^{+} \mathrm{CD} 140 \mathrm{a}^{+}$stromal cells, which have only limited CFU-F activity (Figure 1A). ${ }^{1}$ In addition, EGR1 expression was significantly higher in steady-state adult BMSC (CD31-CD271 ${ }^{+}$) in comparison to fetal BMSC, BMSC in regenerating marrow, and BM endothelial cells $\left(\mathrm{CD} 1^{+} \mathrm{CD}^{+}\right)$(Figure $\left.1 \mathrm{~B}\right)$. None of the other EGR transcription factor family members were expressed at comparable levels in BMSC or endothelial cells (Online Supplementary Figure S1A and B).

\section{Upregulation of EGR1 increases BMSC cell hematopoietic stroma-support}

Motivated by the selective high expression of EGR1 in hematopoiesis-supporting BMSC, we went on to investigate the functions of EGR1 employing gain-of-function and loss-of-function approaches.

Effective reduction of EGR1 expression in BMSC was induced by two (shEGR1-1 and shEGR1-4) of four tested EGR1-specific shRNA (Online Supplementary Figure S2A). EGR1 overexpression in BMSC by more than two-fold was realized using the lentiviral vector encoding full length human EGR1 (Online Supplementary Figure S2B).

BMSC cell hematopoietic stroma supporting function was evaluated by co-culturing CB CD $34^{+}$cells with EGR1 knockdown stromal cells, EGR1 over-expressing stromal cells and corresponding controls, respectively, as adherent feeder cells. Total numbers of hematopoietic cells increased when $\mathrm{CD}_{34}{ }^{+}$cells were expanded on stroma feeder layers compared to cultures without stroma support, but no differences were observed between the different stromal cell groups (Figure 2A and B). However, EGR1 knockdown feeder cells failed to effectively support the $\mathrm{CD}_{34}{ }^{+}$phenotype while the EGR1 over-expressing stromal cells efficiently maintained this population 
(Figure 2C). Furthermore, although not significant, both percentages and absolute numbers of transplantable $\mathrm{CD} 34^{+} \mathrm{CD} 90^{+}$cells were reduced when $\mathrm{CD} 34^{+}$cells were co-cultured on EGR1 knockdown stromal cells $(1.76 \pm 0.76$ fold for shEGR1 vs. $2.64 \pm 1.47$ fold for scramble control compared with no stroma control), whereas the production of $\mathrm{CD} 34^{+} \mathrm{CD} 90^{+}$cells was significantly increased with EGR1 over-expressing stroma feeder cells (6.97 \pm 4.53 -fold) compared to controls (3.11 \pm 2.65 -fold) (Figure 2D). Accordingly, transplantation into NSG mice demonstrated that $\mathrm{CB}$ CD34 $4^{+}$cells expanded on EGR1 over-expressing stroma exhibited higher long-term in vivo reconstitution levels with stable lymphoid and myeloid lineage engraftment compared with controls and EGR1 knockdown feeder cells (Figure 2E-G). These data therefore clearly indicated that EGR1 expression regulates the hematopoietic stroma support function of BM stromal cells.

\section{EGR1-induced enhanced hematopoietic support is mediated through soluble factors and cell-cell interaction}

Cord blood $\mathrm{CD}^{+} 4^{+}$expansion experiments using transwell cultures showed that numbers and percentages of ex vivo expanded $\mathrm{CD} 34^{+}$cells and $\mathrm{CD} 34^{+} \mathrm{CD} 90^{+}$as well as total nucleated cells were reduced in all transwell cocultures compared to stroma-contact conditions (Figure 3A-C and Online Supplementary Figure S3). Reductions were even more drastic in cultures stimulated with conditioned media from EGR1 knockdown and over-expressing cell cultures, respectively, instead of feeder cells. Numbers of total CD34+ and $\mathrm{CD} 34^{+} \mathrm{CD} 90^{+}$cells generated were highest in the EGR1 overexpression groups, both in standard and transwell co-cultures, but also in nonstroma cultures stimulated by conditioned medium collected from EGR1 over-expressing stromal cells. These data thus indicated that EGR1 effects were both cell-cell contact and soluble factor-mediated, and confirmed the role of EGR1 in hematopoietic support regulation.
Stromal EGR1 induces expression of hematopoietic supporting genes that contribute to preserve primitive phenotype of $\mathrm{CD} 34^{+}$cells

To further explore the mechanism of EGR1-mediated hematopoiesis support, an array-based gene expression analysis was performed comparing EGR1 cells and control cells. In total, 189 genes were significantly up-regulated in EGR1 over-expressing cells, including genes involved in signal transduction, as well as adhesion molecule and cytokine genes (Online Supplementary Table S1 and Online Supplementary Figure S4A). Six of the up-regulated genes (CCL28, VCAM1, TIMP3, TNC, ENPP2 and MFAP4) (Figure 4A) have been reported to be genes supporting hematopoiesis, ${ }^{9-14}$ and we chose to further investigate CCL28 and VCAM1 as representatives for soluble factors and surface expressed, cell-cell contact mediating molecules, respectively.

First, CCL28 levels secreted by stromal cells in culture were assessed. CCL28 levels were higher in cultures with EGR1 over-expressing cells compared to controls (Figure 4B). Exogenous CCL28 enhanced the expansion of functional progenitor cells in co-cultures with EGR1 knockdown stroma feeder cells (Figure 4D-F and Online Supplementary Figure S4B-D). Furthermore, ex vivo expansion of $\mathrm{CD} 34^{+}$and $\mathrm{CD} 34^{+} \mathrm{CD} 90^{+}$cells in co-culture with EGR1 over-expressing stroma was dramatically inhibited by CCL28 blocking antibody in both standard medium and cytokine-free conditions (Figure 4G-I and Online Supplementary Figure S4E-G).

VCAM1 was slightly up-regulated in EGR1 overexpressing cells (Figure 4C). Functionally, the production of $\mathrm{CD} 34^{+}$and $\mathrm{CD} 34^{+} \mathrm{CD} 90^{+}$cells in EGR1 over-expressing BMSC co-cultures was dramatically reduced when VCAM1 was blocked by a neutralizing antibody. Expansion rates were further reduced when CCL28 and VCAM1 blocking antibodies were used concurrently (Figure 4G-I and Online Supplementary Figure S4E-G).

Taken together, these data indicated that EGR1 overexpression enhanced BMSC stroma supporting capacity and,
A

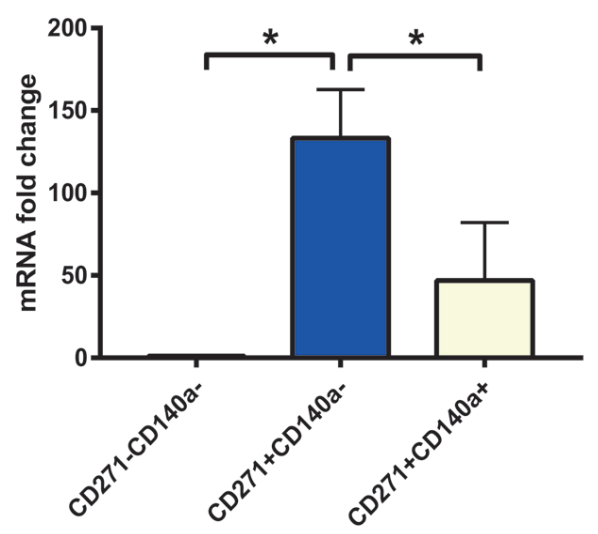

B
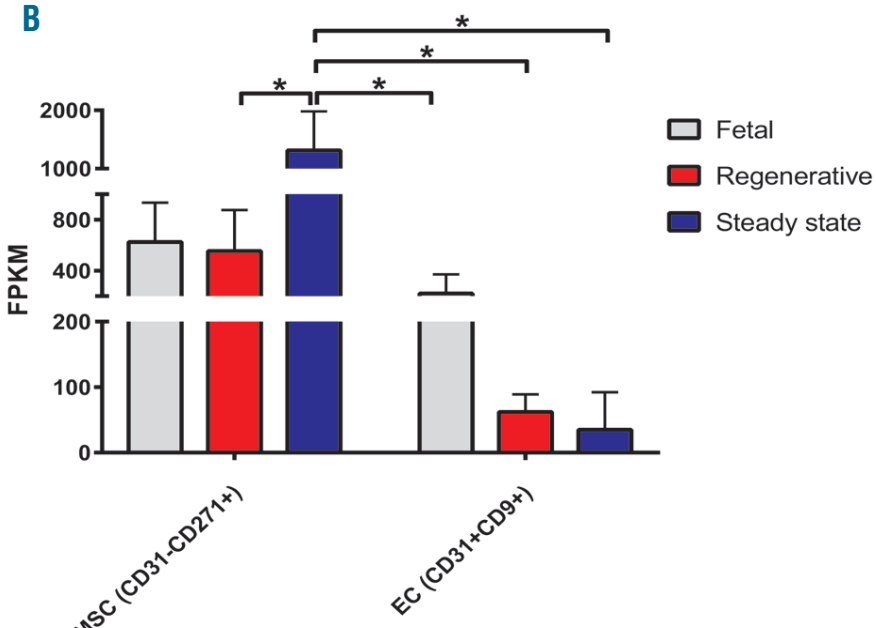

Figure 1. EGR1 is highly expressed by primary bone marrow mesenchymal stromal cells (BMSC). (A) Quantitative real-time polymerase chain reaction (rtPCR) of

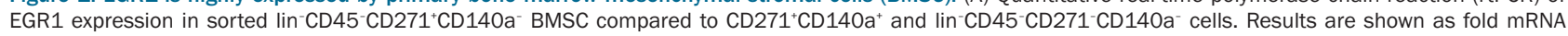
change after standardizing with GAPDH levels. (B) Transcript analysis by massive parallel RNA sequencing of EGR1-4 expression in BMSC (CD31-CD271+) isolated from human fetal, regenerative and steady-state BMSC compared to CD31+CD9+endothelial cells (EC). FPKM: fragments per kilobase million. Data are shown as mean \pm standard deviation, $\mathrm{n}=3-10$. * $P<0.05$. 
consequently, improved ex vivo expansion of hematopoietic cells by up-regulating a panel of hematopoiesis-supporting genes, including cytokines and cell surface expressed molecules such as CCL28 and VCAM1.

\section{EGR1 knockdown induces bone marrow stromal cell proliferation mediated by elevated reactive oxygen species}

EGR1 functions either as a cell cycle promoter or a cell proliferation inhibitor depending on the cell type and the environment. We therefore investigated how EGR1 expression impacted BMSC proliferation. Our data showed that EGR1 knockdown BMSC exhibited higher percentages of dividing cells, shorter population doubling times, and enhanced colony-forming capacities (Figure 5A-C and Online Supplementary Figure S5A). On the other hand, CFU-F activity was virtually absent in EGR1 overexpressing cells and population doubling times were sub-

A

no stroma

scr ctr

ShEGR1-1

GFP ctr

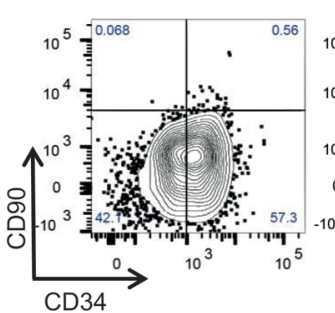

B

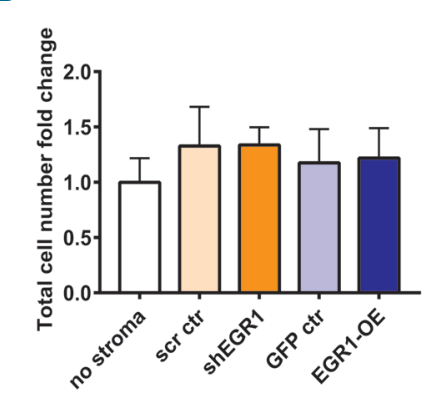

C

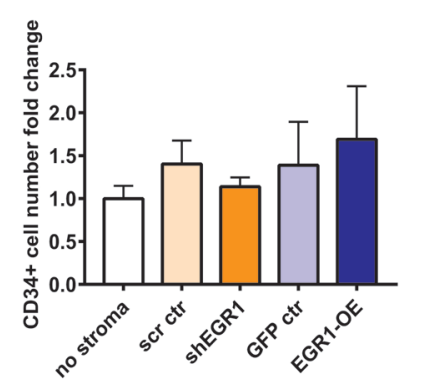

D

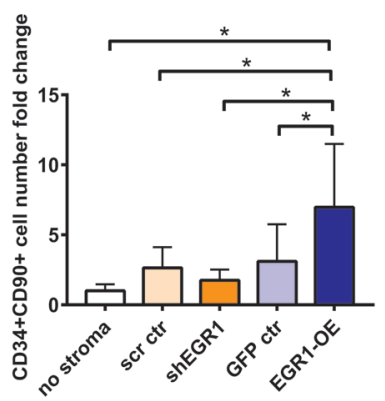

E

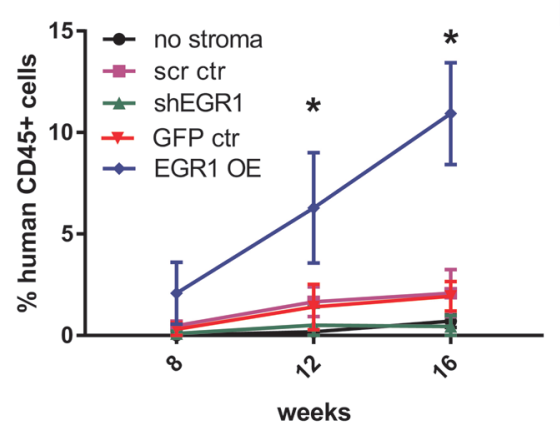

F



G no stroma
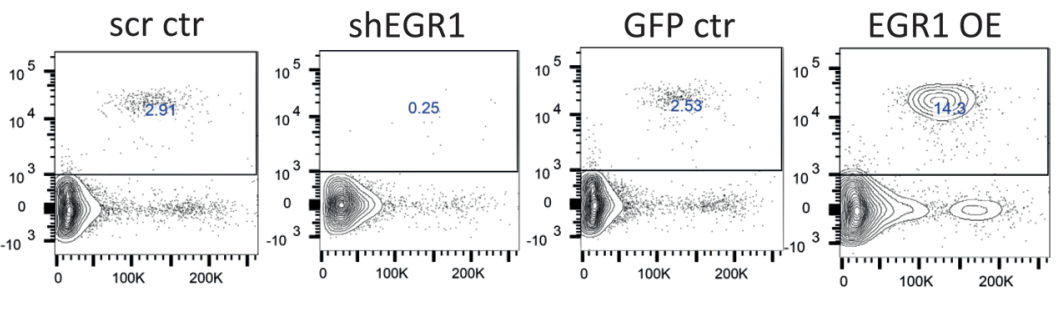

Figure 2. Increased EGR1 expression in mesenchymal stromal cells (MSC) promotes the ex vivo expansion of transplantable cord blood CD34 ${ }^{+}$cells. Five thousand cord blood CD34+ cells were co-cultured for four days on a feeder layer of 10,000 BMSC transfected with scramble control, shEGR1, green fluorescent protein control (GFP ctr) and EGR1 overexpression plasmids, respectively, in SFEM supplemented with $25 \mathrm{ng} / \mathrm{mL}$ of SCF, TPO and FIt3L (STF25). (A) Representative FACS profiles of co-culture generated cells are shown. The type of feeder cells is indicated on top of the respective FACS plot. (B-D) Fold change of total number of hematopoietic cells $(B), C D 34^{+}$cells $(C)$, and $C D 34^{+}{ }^{2} D 90^{+}$cells (D) produced after four days in culture. Results are shown as fold change relative to the cell number of standard $\mathrm{CD}^{+}{ }^{+}$culture (STF25) without stroma support. $\mathrm{N}=9-12$. ${ }^{*} \mathrm{P}<0.05$. (E-G) Analysis of human hematopoietic cell engraftment following intravenous transplantation of the culture equivalent (4 days culture in SFEM, STF25) of 50,000 input CD34 cells into NSG mice. Human engraftment was assessed 8 , 12 and 16 weeks after transplantation by flow cytometry using human-specific antibodies against CD45, CD15/CD33/CD66b and CD19. *P<0.05 between EGR1 OE and each of the other groups. (E) Percentage of human $\mathrm{CD}_{4} 5^{+}$cells in the peripheral blood of NSG mice at different time points after intravenous transplantation. Data represent the mean \pm standard deviation (SD) of a total of 4-5 mice per time point except no stroma control, which shows the data for two mice. (F) Lineage distribution of human cells (mean $\pm S D, n=2-5$ ) and $(G)$ representative FACS plots 16 weeks after transplantation. 
stantially increased accordingly (Figure 5B and C).

Proteomic analysis was performed to investigate the mechanisms behind the EGR1 knockdown-induced increased proliferation. Of a total of 4,520 identified proteins, 190 were differentially expressed between EGR1 knockdown stromal cells and scramble and non-transfected controls (Online Supplementary Tables S4 and S5 and Online Supplementary Figure S4E-G). A group of ten proteins (HSD17B11, UQCRC1, CYP1B1, NDUFA8, TXNDC17, CYCS, FADS3, PDIA5, PCYOX1, OSOX1) related to oxidative-reduction processes was expressed lower in EGR1 knockdown cells (Figure 6A and B) as were GLS1, GPX1 and GSTP1, which are also associated with intracellular reactive oxygen species (ROS) accumulation. ${ }^{15-17}$ Accordingly, ROS levels in EGR1 knockdown cells were increased compared to controls (Figure 6C and D).

Inhibiting ROS using an antioxidant cocktail (L-ascorbic acid and citric acid), N-acetylcysteine (NAC), or apocynin considerably reduced ROS production in EGR1 knock- down cells (Figure 6E). ROS reduction caused a decrease in the fraction of dividing EGR1 knockdown cells (Figure $6 \mathrm{~F})$ and in the numbers of CFU-F with complete abrogation of colony formation when using the antioxidant cocktail (Figure 6G). In contrast, neither myeloperoxidase (MPO) inhibitor 4-ABAH nor the small molecule inhibitor LY2228820 (Figure 6E-G) affected ROS levels, percentages of proliferating cells or CFU-F numbers.

Consistent with the increased proliferation in EGR1knockdown BMSC, gene expression profiling identified a group of down-regulated genes involved in cell death and apoptosis (BFAR, EIF4G2, TSPO, RABEP1, MCL1, TNFRSF12A, TBRG4, MYC, DDIT4) in EGR1 knockdown cells (Online Supplementary Table S2 and Online Supplementary Figure $S 5 B$ and C). Furthermore, positive regulators of cell proliferation and negative regulators of apoptosis were down-regulated in EGR1 over-expressing cells (Online Supplementary Table S3 and Online Supplementary Figure S5D), which is consistent with the
A

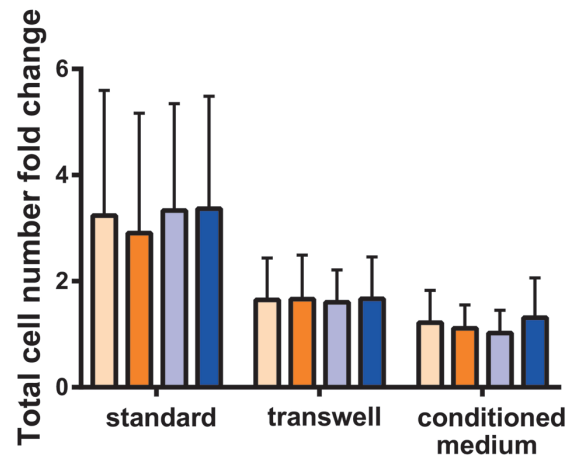

B

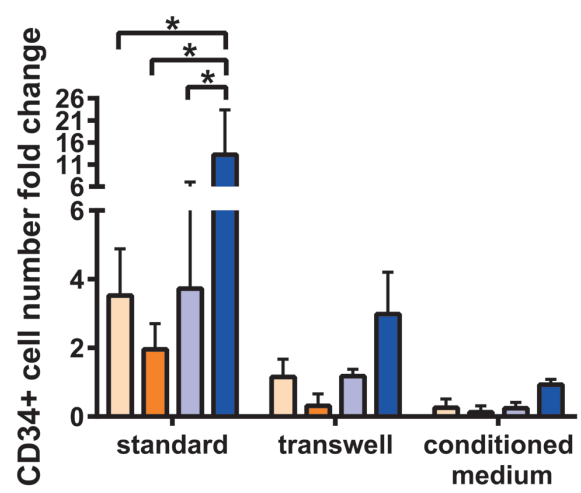

C

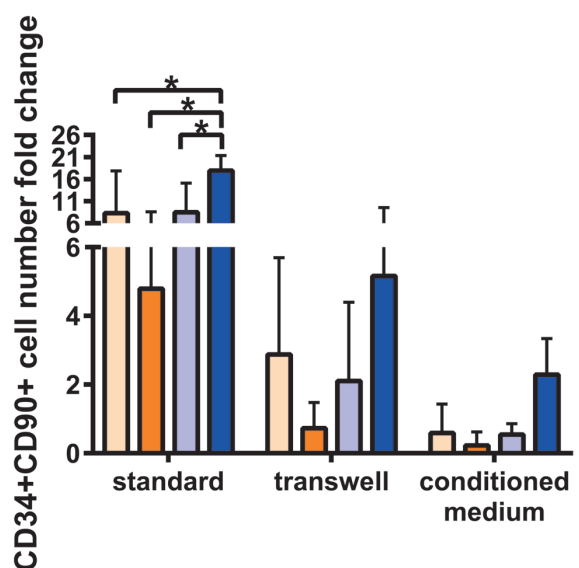

Figure 3. EGR1-induced ex vivo expansion of $\mathrm{CB}$ CD $34^{+}$cells is mediated by both soluble and membrane-bound factors. Five thousand cord blood (CB) $\mathrm{CD} 34^{+}$cells were co-cultured for four days with 10,000 feeder bone marrow mesenchymal stromal cells (BMSC) transfected with scramble control, shEGR1, green fluorescent protein control (GFP ctr) and EGR1 overexpression plasmids, respectively, in serum-free expansion medium supplemented with $25 \mathrm{ng} / \mathrm{mL}$ of SCF, TPO and Flt3L. Co-cultures were performed in either standard culture plates (standard) or transwell culture plates with the stromal cells in the bottom well and $\mathrm{CD}_{4} 4^{+}$cells in the insert (transwell). For conditioned medium cultures, 10,000 BM-derived stromal cells transfected with scramble control, shEGR1, GFP control and EGR1 overexpression plasmids, respectively, were cultured with $200 \mu \mathrm{L}$ serum-free expansion medium supplemented with $25 \mathrm{ng} / \mathrm{mL}$ of SCF, TPO and Flt3L for four days. Conditioned media were collected and used to stimulate cultures with $\mathrm{CB} C \mathrm{CD} 34^{+}$cells (without feeder cells). Fold change of total cell number (A), cell number of $\mathrm{CD} 34^{+}$cells $(\mathrm{B})$ and $\mathrm{CD} 34^{+} \mathrm{CD} 90^{+}$cells (C) produced after four days in culture are shown as mean+standard deviation. Three independent experiments were performed with cells from different donors. Representative results are shown for one of the experiments, $* P<0.05$. 
reduced proliferation of EGR1 over-expressing cells.

These data therefore indicate that increased proliferation of EGR1 knockdown cells is, at least partly, mediated by increased ROS levels and downregulation of cell death genes, whereas downregulation of cell proliferation and apoptosis inhibiting genes was observed upon EGR1 overexpression. Thus, whereas increased EGR1 expression improved the stroma supporting function of BMSC, cell proliferation was substantially inhibited, indicating a dual role of EGR1 in the regulation of BMSC growth and function. On the other hand, other BMSC functions, such as multi-differentiation capacity (adipogenic, osteogenic and chondrogenic) and surface marker expression profile of BMSC were not affected by changes in EGR1 expression (Online Supplementary Figure S6A and B).

\section{Discussion}

Bone marrow is the major site of residence of both HSC and BMSC. The most crucial functions of BMSC are to maintain the turnover of the BM stroma and skeletal tissues and to provide critical hematopoietic support. However, the mechanisms that regulate these different
A



B

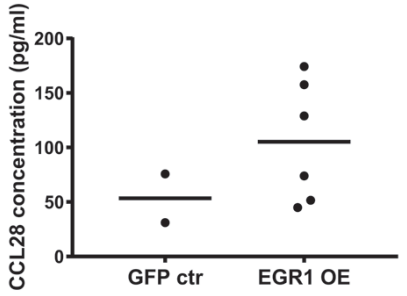

C

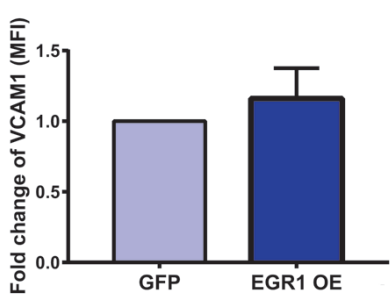

D

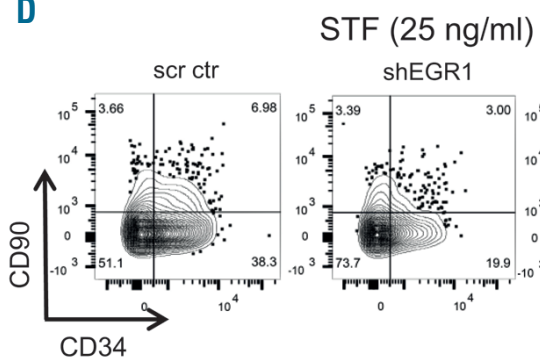

E



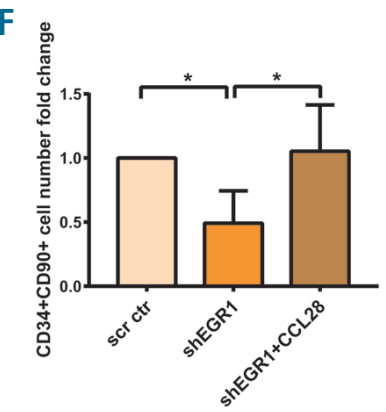

G

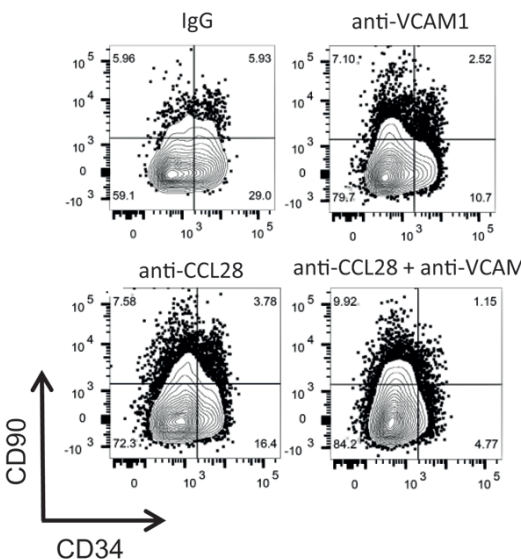

H

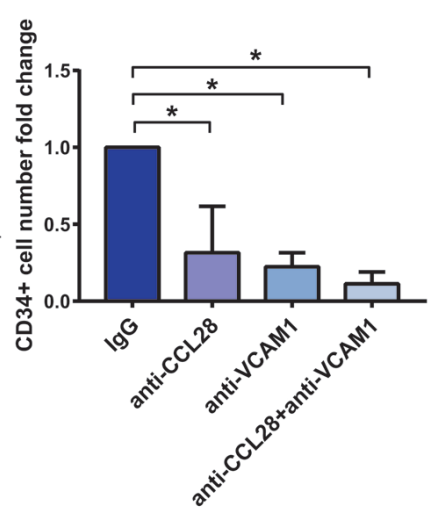

I

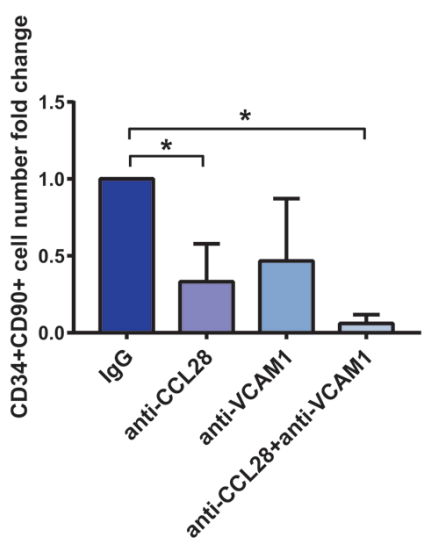

Figure 4. Hematopoiesis-supporting genes are up-regulated in EGR1 over-expressing mesenchymal stromal cells (MSC). (A) Heatmap of significantly up-regulated hematopoietic supporting genes in EGR1 overexpression cells versus controls $(n=4)$. (B) Secreted CCL28 concentrations in cell culture supernatants of EGR1 overexpressing bone marrow stromal cells (BMSC) (EGR1 OE) and green fluorescent protein control (GFP ctr) ( $n=2-6)$. (C) Fold change of surface expression of VCAM1 (CD106) in EGR1 over-expressing cells compared with GFP control cells. VCAM1 expression is shown as fold change of the geometric mean fluorescence intensity (MFI) after standardizing with GFP control cells (n=3-4). (D-F) 5,000 cord blood CD34+ cells were co-cultured for four days with 10,000 BM-derived feeder stromal cells transfected with scramble control and shEGR1 plasmids, respectively, in cytokine-free or standard STF25 culture conditions supplemented with or without 100 $\mathrm{ng} / \mathrm{mL}$ CCL28. Standard culture (STF25): SFEM supplemented with $25 \mathrm{ng} / \mathrm{mL}$ of SCF, TPO and Flt3L ( $\mathrm{n}=3$ ). (D) Representative FACS profiles of co-culture generated cells in standard culture. The type of feeder cells is indicated on top of the FACS plots. Fold change of total numbers of CD34 ${ }^{+}$cells and CD34 ${ }^{+} \mathrm{CD} 90^{+}$cells produced in standard STF25 cultures (E and F). (G-I) 5,000 cord blood CD34+ cells were co-cultured for four days with 10,000 EGR1 overexpression cells as feeder cells in standard culture media supplemented with neutralizing antibody against CCL28, VCAM1 and IgG control (all at $100 \mathrm{ng} / \mathrm{mL}$ ) for four days. (G) Representative FACS profiles of co-culture generated cells. Total number of CD34+ cells $(\mathrm{H})$ and CD34+CD90+ cells (I) produced in the co-cultures without/with neutralizing antibodies as indicated by the $x$-axis labels $(n=3) . * P<0.05$ 
functions are not well known. Murine studies using inducible gene deletion strategies in BMSC indicated a tight control of key BMSC functions to maintain homeostasis of the hematopoietic and the skeletal system. ${ }^{18,19}$ Here we report for the first time that EGR1 is a key regulator in human BMSC.

EGR1 is a member of the immediate early response transcription factor family with a role in regulating development, growth control and stress response in many tissues. Based on our EGR1 expression data in primary BMSC, ${ }^{1}$ and information on the role of EGR1 in stromal cell growth factor production regulation and hematopoietic stem cell regulation, ${ }^{6,20}$ we hypothesized that EGR1 could also be an important regulator of BM stromal stem cells.

In accordance with our previous data ${ }^{1}$ we found that EGR1 expression was substantially higher in highly CFUF-enriched primary $\operatorname{lin}^{-} \mathrm{CD} 45^{-} \mathrm{CD} 271^{+} \mathrm{CD} 140 \mathrm{a}^{-} \mathrm{BMSC}$ (Figure 1A). In addition, EGR1 was significantly higher expressed in steady-state adult BMSC in comparison to fetal BMSC and BMSC in regenerating marrow (Figure 1B), which have higher proliferation rates as indicated by high cell cycle activator expression levels in fetal BMSC (data not shown) and previously reported proliferation data. $^{21,22}$ Moreover, EGR1 was the only member of the EGR family (EGR1-4) that was highly expressed in BMSC (Online Supplementary Figure $S 1 A$ and B), in contrast to other cell types that also co-express other EGR family members. ${ }^{23,24}$

These data implied that EGR1 expression levels might be connected to the regulation of key BMSC functions, i.e. hematopoietic support in steady-state and stroma proliferation in situations with regenerative demands. In fact, EGR1 overexpression increased BMSC hematopoietic stroma-supporting function and facilitated the effective generation of transplantable hematopoietic stem cells, while EGR1 knockdown abrogated the stroma contribution in HSC expansion co-cultures (Figure 2). Furthermore, a clear reduction in ex vivo expanded HSC was recorded in transwell cultures and when using conditioned medium (Figure 3). Our data thus indicated that EGR1 regulated BMSC stroma support functions mediated by both cell-cell contact and soluble factors, both of which are required to realize effective ex vivo HSC expan- sion. ${ }^{25-28}$ Accordingly, gene array expression profiling identified hematopoiesis-supporting genes as EGR1 downstream targets, which corresponded to both secreted and surface-expressed molecules (Figure 4A). Of these, we investigated the functional role of CCL28 and VCAM1 as examples for a potent niche-secreted soluble growth factor $^{11}$ and an adhesion molecule, respectively. Here, CCL28 supplementation and blocking experiments (Figure 4D-I) clearly indicated a contributing role of CCL28 in EGR1mediated ex vivo expansion of $\mathrm{CB} \mathrm{CD}_{3} 4^{+}$cells on EGR1 over-expressing stromal cells. Similarly, the role of VCAM1 was demonstrated by blocking VCAM1 with neutralizing antibodies (Figure 4G-I).

Successful ex vivo expansion of HSC represents a promising approach to provide sufficient numbers of transplantable stem cells and to facilitate the development of cell and gene therapies. A number of approaches to expand HSC have been pursued, including enhancing positive signals and inhibiting negative signals for HSC selfrenewal. ${ }^{29,30}$ Our results indicate that modification of feeder cell regulation by EGR overexpression represents a novel approach to generate an optimized microenvironment that supports HSC self-renewal and growth with the potential to improve current HSC expansion culture conditions. Furthermore, one can envision strategies to increase EGR1 expression in vivo to improve hematopoietic stromal support function, for example, in transplantation patients with poor graft function, and possibly even in patients with insufficient hematopoiesis, such as lowrisk myelodysplastic syndrome.

The effect of EGR1 on cell proliferation depends on the biological context. ${ }^{31,32}$ Our data clearly demonstrated that high expression of EGR1 inhibited proliferation and colony-formation of human BMSC, whereas reduced EGR1 levels caused the opposite effects (Figure 5). Thus, EGR1 expression modulation might not only be used to modify BMSC stroma support function, but could also be utilized to increase BMSC proliferation in vitro to optimize BMSC expansion strategies, and possibly also in vivo to induce stroma and bone tissue growth.

Proteomic analysis of EGR1 knockdown cells identified a group of down-regulated proteins related to oxidativereduction processes. Accordingly, increased ROS levels were observed in EGR1 knockdown cells (Figure 6C),
A

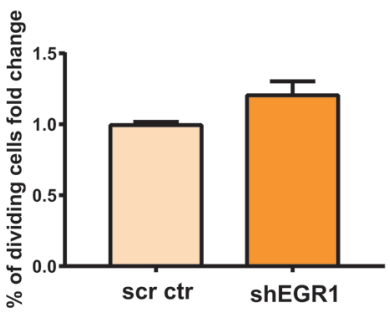

B

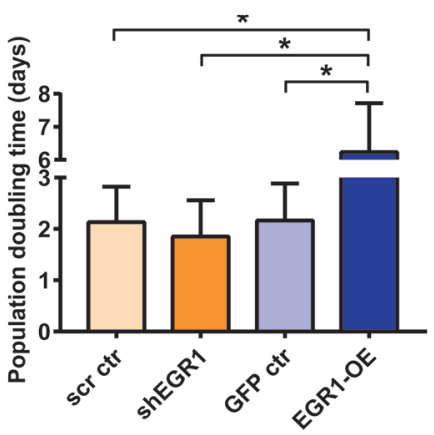

c

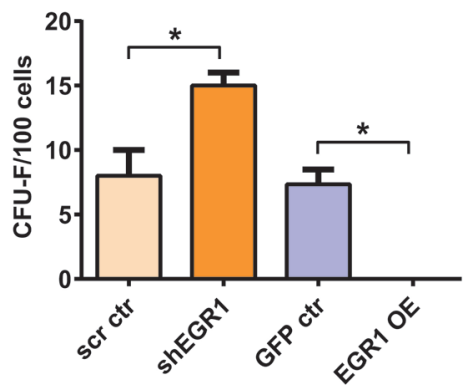

Figure 5. Proliferation of EGR1 knockdown bone marrow mesenchymal stromal cells (BMSC) is increased. (A) Percentage of dividing cells in EGR1 knockdown BMSC in comparison with scramble control. Data are shown as mean \pm standard deviation (SD) $(n=5)$ for passage $2-5$ cells. (B) Population doubling times of passage 2-5 BMSC transfected with scramble control, shEGR1, green fluorescent protein control (GFP ctr) and EGR1 overexpression plasmids, respectively. Data are shown as mean $\pm S D(n=3)$. (C) Frequencies of colony-forming units-fibroblasts (CFU-F) in BM-derived MSC (passage 2-5) transfected with scramble control, shEGR1, GFP ctr and EGR1 overexpression plasmids, respectively. Data are presented as mean $\pm \operatorname{SD}(n=3)$. $* P<0.05$. 
which have been reported to promote cell proliferation, differentiation and survival. ${ }^{33-35}$ Furthermore, experiments using different ROS inhibitors showed that ROS production in BMSC was mostly affected by oxidative pathway changes, and to a lesser extent by p38 pathway alterations or 4-ABAH induced inhibition of myeloperoxidase (MPO).
In addition to the role of EGR1 in regulating key BMSC functions in vitro as demonstrated here, we observed that EGR1 expression levels were significantly different in different (patho)physiological situations. These expression data were generated with prospectively isolated BMSC and can therefore be considered to accurately reflect the in vivo situation. We found that EGR1 was significantly
A

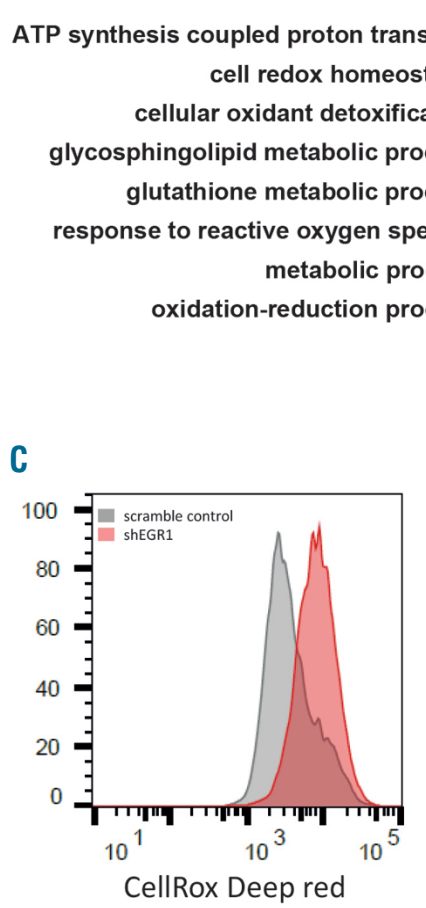

B



E

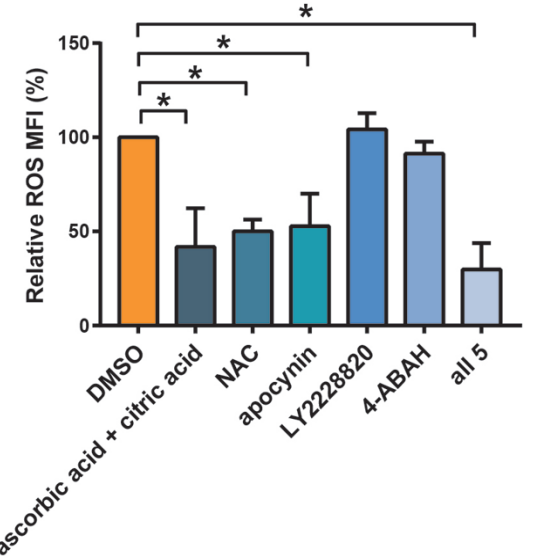

$\mathbf{F}$

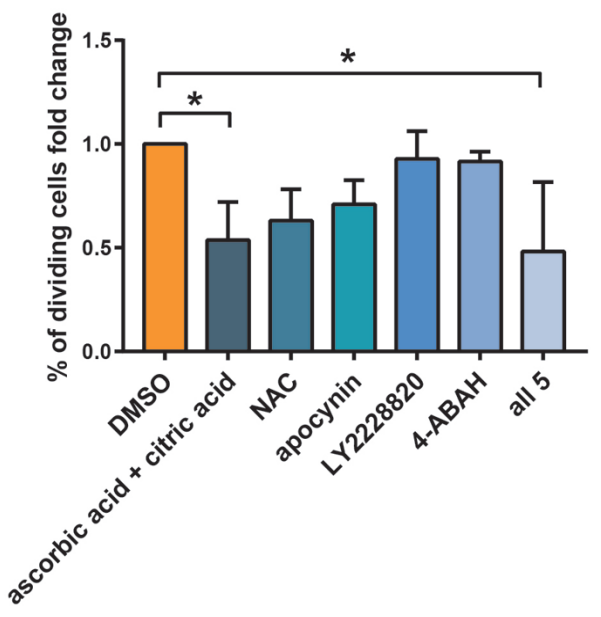

G

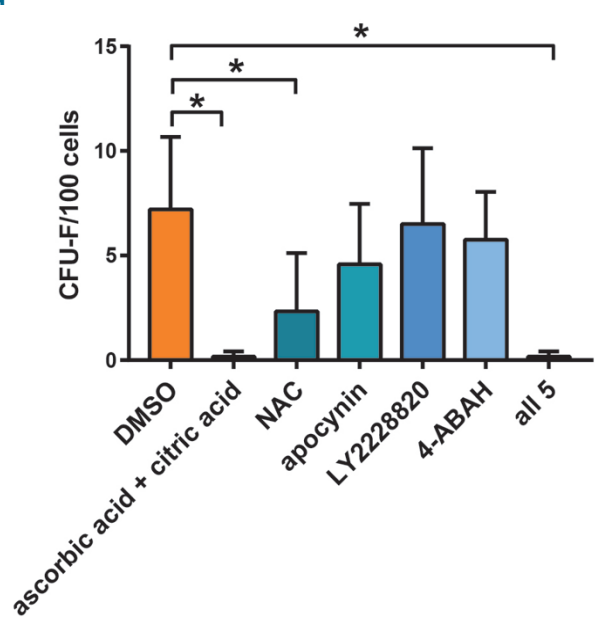

Figure 6. Increased proliferation in EGR1 knockdown bone marrow mesenchymal stromal cells (BMSC) is mediated by reactive oxygen species (ROS). (A) Biological process annotations for down-regulated proteins in EGR1 knockdown cells were identified using the DAVID Bioinformatics Resources 6.8. (B) Heatmap of down-regulated proteins related to oxidative-reduction processes in EGR1 knockdown cells versus controls. NT: non-transfected cells; scr ctr: scramble control. (C) Representative FACS histogram of ROS levels in EGR1 knockdown cells compared with scramble control. (D) Fold change of ROS in EGR1 knockdown cells in comparison with scramble control. Data are shown as mean fluorescence intensity (MFI) fold change \pm standard deviation (SD) $(n=3)$. ROS MFI levels relative to dimethy sulfoxide (DMSO) controls (E), percentage of dividing cells expressed as fold change compared to DMSO controls (F), and colony-forming units-fibroblast (CFU-F) frequencies $(\mathrm{G})$ in EGR1 knockdown cells after ROS inhibitor treatment. Data are shown as mean $\pm \mathrm{SD}(\mathrm{n}=3)$. NAC: $N$-acetylcysteine. $* P<0.05$. 
higher expressed in adult steady-state BMSC compared with fetal BMSC and BMSC in regenerating marrow (Figure 1B), both of the latter showing higher proliferation compared to steady-state BMSC. Thus, these data point to a possible key role in vivo of EGR1, regulating the response to the different requirements in development and under pathological conditions. In the fetal period, there is demand for a high proliferation of BMSC with potent osteogenic and stroma differentiation potential. Likewise, primarily cellular regeneration is required after stromal damage, for example, following myelotoxic chemo- or radiotherapy. In addition, murine data demonstrated that BMSC expanded considerably following irradiation and BM transplantation. ${ }^{22}$ In contrast, hematopoietic support is most important in steady-state adult BM, whereas there is only a limited need for BMSC proliferation. However, whether or not a demand-controlled EGR1 regulation of proliferation and stroma function is operative in vivo has not yet been investigated, but is certainly an interesting and highly relevant topic for future research.

Taken together, we demonstrate that EGR1 is a key dual regulator of human BMSC which controls a genetic program co-ordinating specific functions of BMSC in their different biological contexts. High EGR1 expression induced potent hematopoietic stroma support by expression of high levels of hematopoiesis-supporting genes and suppressed BMSC proliferation, whereas EGR1 downregulation promoted BMSC proliferation at the expense of hematopoietic support function. These data thus considerably expand our current understanding of the regulation of BMSC under distinct physiological and developmental conditions. Furthermore, they have clear implications for the development of stroma replacement and repair strategies, for example, for optimizing BMSC expansion protocols in vitro and to improve stroma function in transplantation patients with poor graft function, as well as stroma-mediated HSC expansion approaches.

\section{Funding}

This work was supported by funds from the HematoLinne and StemTherapy Program, the Swedish Cancer Foundation, the Swedish Childhood Cancer Foundation, Gunnel Björk's Testament, Gunda Nilsson's Testament, the Ake och Inger Bergkvists stiftelse, ALF (Government Public Health Grant), and the Skane County Council Research Foundation.

\section{Acknowledgments}

The authors would like to thank Helene Larsson and Anna Jonasson for their help to collect bone marrow samples and the Lund Stem Cell Center FACS facility personnel for technical assistance. Furthermore, support from the Swedish National Infrastructure for Biological Mass Spectrometry (BioMS) is gratefully acknowledged. We would also like to thank Professors Axel Schambach, Manuel Grez, and Thomas Moritz for providing the A2UCOE-EFS promoter used to generate the lentivector driving expression of EGR1, and Jonas Larsson for crtically reading the manuscript.

\section{References}

1. Li $\mathrm{H}$, Ghazanfari $\mathrm{R}$, Zacharaki $\mathrm{D}$, et al. Low/negative expression of PDGFR-alpha identifies the candidate primary mesenchymal stromal cells in adult human bone marrow. Stem Cell Reports. 2014;3(6):965-974.

2. Tormin A, Li O, Brune JC, et al. CD146 expression on primary nonhematopoietic bone marrow stem cells is correlated with in situ localization. Blood. 2011; 117(19):5067-5077

3. Christy BA, Lau LF, Nathans D. A gene activated in mouse 3 T3 cells by serum growth factors encodes a protein with "zinc finger" sequences. Proc Natl Acad Sci U S A. 1988; 85(21):7857-7861.

4. Milbrandt J. A nerve growth factor-induced gene encodes a possible transcriptional regulatory factor. Science. 1987;238(4828):797799.

5. Lemaire P, Revelant O, Bravo R, Charnay P. Two mouse genes encoding potential transcription factors with identical DNA-binding domains are activated by growth factors in cultured cells. Proc Natl Acad Sci U S A. 1988;85(13):4691-4695.

6. Min IM, Pietramaggiori G, Kim FS, Passegue E, Stevenson KE, Wagers AJ. The transcription factor EGR1 controls both the proliferation and localization of hematopoietic stem cells. Cell Stem Cell. 2008;2(4):380-391.

7. Reumann MK, Strachna O, Lukashova L, et al. Early growth response gene 1 regulates bone properties in mice. Calcif Tissue Int. 2011;89(1):1-9.

8. Kenswil KJG, Jaramillo AC, Ping Z, et al.
Characterization of Endothelial Cell Associated with Hematopoietic Niche Formation in Humans Identifies IL-33 As an Anabolic Factor. Cell Rep. 2018;22(3):666-678.

9. Zhang CC, Kaba M, Ge G, et al Angiopoietin-like proteins stimulate ex vivo expansion of hematopoietic stem cells. Nat Med. 2006;12(2):240-245

10. Nakajima H, Ito M, Smookler DS, et al. TIMP-3 recruits quiescent hematopoietic stem cells into active cell cycle and expands multipotent progenitor pool. Blood. 2010;116(22):4474-4482.

11. Karlsson C, Baudet A, Miharada N, et al. Identification of the chemokine CCL28 as a growth and survival factor for human hematopoietic stem and progenitor cells. Blood. 2013;121(19):3838-3842.

12. Nakamura-Ishizu A, Okuno Y, Omatsu Y, et al. Extracellular matrix protein tenascin$\mathrm{C}$ is required in the bone marrow microenvironment primed for hematopoietic regeneration. Blood. 2012;119(23):5429-5437.

13. Li H, Yue R, Wei B, Gao G, Du J, Pei C. Lysophosphatidic acid acts as a nutrientderived developmental cue to regulate early hematopoiesis. EMBO J. 2014;33(12):1383 1396.

14. Mazo IB, Massberg S, von Andrian UH. Hematopoietic stem and progenitor cell trafficking. Trends Immunol. 2011; 32(10):493-503

15. Kanwal R, Pandey M, Bhaskaran N, et al. Protection against oxidative DNA damage and stress in human prostate by glutathione S-transferase P1. Mol Carcinog. 2014;53(1):8-18.
16. Okazaki A, Gameiro PA, Christodoulou D et al. Glutaminase and poly(ADP-ribose) polymerase inhibitors suppress pyrimidine synthesis and VHL-deficient renal cancers. J Clin Invest. 2017;127(5):1631-1645.

17. Dokic I, Hartmann C, Herold-Mende C, Regnier-Vigouroux A. Glutathione peroxidase 1 activity dictates the sensitivity of glioblastoma cells to oxidative stress. Glia. 2012;60(11):1785-1800

18. Omatsu Y, Seike M, Sugiyama T, Kume T, Nagasawa T. Foxc1 is a critical regulator of haematopoietic stem/progenitor cell niche formation. Nature. 2014;508(7497):536540.

19. Seike M, Omatsu Y, Watanabe H, Kondoh G, Nagasawa T. Stem cell niche-specific Ebf3 maintains the bone marrow cavity. Genes Dev. 2018;32(5-6):359-372

20. Kerpedjieva SS, Kim DS, Barbeau DJ Tamama K. EGFR ligands drive multipotential stromal cells to produce multiple growth factors and cytokines via early growth response-1. Stem Cells Dev. 2012; 21(13):2541-2551.

21. Guillot PV, Gotherstrom C, Chan J, Kurata $\mathrm{H}$, Fisk NM. Human first-trimester fetal MSC express pluripotency markers and grow faster and have longer telomeres than adult MSC. Stem Cells. 2007;25(3):646-654

22. Abbuehl JP, Tatarova Z, Held W, Huelsken J. Long-term engraftment of primary bone marrow stromal cells repairs niche damage and improves hematopoietic stem cell transplantation. Cell Stem Cell. 2017; 21(2):241-255.e6.

23. Shao H, Kono DH, Chen LY, Rubin EM Kaye J. Induction of the early growth 
response (Egr) family of transcription factors during thymic selection. J Exp Med. 1997;185(4):731-744.

24. Poirier R, Cheval H, Mailhes C, et al. Distinct functions of egr gene family members in cognitive processes. Front Neurosci. 2008;2(1):47-55

25. Harvey K, Dzierzak E. Cell-cell contact and anatomical compatibility in stromal cellmediated HSC support during development. Stem Cells. 2004;22(3):253-258.

26. Kawada H, Ando K, Tsuji T, et al. Rapid ex vivo expansion of human umbilical cord hematopoietic progenitors using a novel culture system. Exp Hematol. 1999;27(5):904-915.

27. Thiemann FT, Moore KA, Smogorzewska EM, Lemischka IR, Crooks GM. The murine stromal cell line AFT024 acts specifically on human CD34+CD38- progenitors to maintain primitive function and immunophenotype in vitro. Exp Hematol. 1998;26(7):612-619.

28. Breems DA, Blokland EA, Ploemacher RE Stroma-conditioned media improve expansion of human primitive hematopoietic stem cells and progenitor cells. Leukemia. 1997;11(1):142-150.

29. Guo B, Huang $X$, Lee MR, Lee SA, Broxmeyer HE. Antagonism of PPAR gamma signaling expands human hematopoietic stem and progenitor cells by enhancing glycolysis. Nat Med. 2018;24(3):360-367.

30. Talkhoncheh MS, Subramaniam A Magnusson M, Kumar P, Larsson J, Baude A. Transient inhibition of NF-kappaB signaling enhances ex vivo propagation of human hematopoietic stem cells. Haematologica. 2018;103(9):1444-1450.
31. Thiel G, Cibelli G. Regulation of life and death by the zinc finger transcription factor Egr-1. J Cell Physiol. 2002;193(3):287-292.

32. Adamson ED, Mercola D. Egr1 transcription factor: multiple roles in prostate tumor cell growth and survival. Tumour Biol. 2002;23(2):93-102.

33. Kobayashi CI, Suda T. Regulation of reactive oxygen species in stem cells and cancer stem cells. J Cell Physiol. 2012;227(2):421430.

34. D'Autreaux B, Toledano MB. ROS as signalling molecules: mechanisms that generate specificity in ROS homeostasis. Nat Rev Mol Cell Biol. 2007;8(10):813-824.

35. Atashi F, Modarressi A, Pepper MS. The role of reactive oxygen species in mesenchymal stem cell adipogenic and osteogenic differentiation: a review. Stem Cells Dev. 2015;24(10):1150-1163. 